\section{International Scientific Journal Theoretical \& Applied Science}

p-ISSN: 2308-4944 (print) e-ISSN: 2409-0085 (online)

Year: $2016 \quad$ Issue: $10 \quad$ Volume: 42

Published: $30.10 .2016 \quad$ http://T-Science.org
Vladimir Igorevich Esaulov
Institute of psychotherapy and clinical psychology

(Moscow),

Psychotherapy Department, Lecturer, Assistant, Department of Psychotherapy, Pirogov Russian National Research Medical

University, Russia v-esaulov@yandex.ru

\title{
THE EXPERIENCE OF THE APPLICATION OF PSYCHOTHERAPEUTIC TECHNIQUES IN THE TREATMENT OF BORDERLINE MENTAL DISORDERS IN PATIENTS WITH IRRITABLE BOWEL SYNDROME
}

\begin{abstract}
This article is dedicated to the analysis of domestic and foreign works on the application of psychotherapy in irritable bowel syndrome (IBS). It is focused on the bio-psycho-social nature of IBS. It pays attention to works focused on comorbid borderline mental disorders and irritable bowel syndrome. The borderline mental disorders are based on various combinations of anxiety and depression. The availability of this comorbid suggests the usage of psychotropic drugs and methods of psychotherapy in treatment of patients with IBS. Most of the studies, dedicated to the application of the psychotherapy in the treatment of patients with IBS, concern the usage of cognitive behavioral therapy techniques, various hypnotic and relaxation techniques. Similarity of the modern psychotherapeutic approach of domestic and foreign researchers, focused on disturbing components of disease, is also described. The own experience of application of psychological techniques in the treatment of borderline mental disorders in patients with IBS is presented. The usage of abdominal version of hypnosis, relaxation techniques and other psychotherapeutic techniques at different stages of the disease is described.

Key words: irritable bowel syndrome, borderline mental disorders, anxiety, depression, psychotherapeutic techniques, hypnosis, cognitive-behavioural therapy, relaxation techniques.

Language: Russian

Citation: Esaulov VI (2016) THE EXPERIENCE OF THE APPLICATION OF PSYCHOTHERAPEUTIC TECHNIQUES IN THE TREATMENT OF BORDERLINE MENTAL DISORDERS IN PATIENTS WITH IRRITABLE BOWEL SYNDROME. ISJ Theoretical \& Applied Science, 10 (42): 77-86.

Soi: http://s-o-i.org/1.1/TAS-10-42-17 Doi: crossef http://dx.doi.org/10.15863/TAS.2016.10.42.17
\end{abstract}

\section{ОБ ОПЫТЕ ПРИМЕНЕНИЯ ПСИХОТЕРАПЕВТИЧЕСКИХ ТЕХНИК В ЛЕЧЕНИИ ПОГРАНИЧНЫХ ПСИХИЧЕСКИХ РАССТРОЙСТВ У ПАЦИЕНТОВ С СИНДРОМОМ РАЗДРАЖЕННОГО КИШЕЧНИКА}

Аннотация: В работе проведен анализ отечественных и зарубежных работ по применению психотерапии при синдроме раздраженного кишечника (СРК). Подчеркнут био-психо-соичиальный характер СРК. Внимание уделено работам, посвященным коморбидности пограничных психических расстройств и синдрома раздраженного кишечника. Основой пограничных психических расстройств являются различные комбинации тревоги и депрессии. Наличие этой коморбидности предполагает введение в лечение больных СРК психотропных препаратов и методов психотерапии. Большинство исследований, посвященных проводимой психотерапии при СРК, касается применения техник когнитивнобихевиоральной терапии, различных гипнотических техник, релаксачионных приемов. В работе отмечена близость современных психотерапевтических приемов, применяемых зарубежными и отечественными исследователи, ориентированных в первую очередь на работу с тревожными составляюшими болезни. Приведен собственный опыт применения психотерапевтических техник в лечении пограничных психических расстройств у пациентов с СРК. Описано применение абдоминального варианта гипноза, релаксачионных техник, и других психотерапевтических техник на разных этапах заболевания.

Ключевые слова: синдром раздраженного кишечника, пограничные психические расстройства, тревога, депрессия, техники психотерапии, гипноз, когнитивно-бихевиоральная терапия, релаксаиионнье приемь

ISPC Computer technologies in science,

Scranton, USA 


\begin{tabular}{|c|c|c|c|c|c|c|}
\hline Impact Factor: & $\begin{array}{l}\text { ISRA (India) } \\
\text { ISI (Dubai, UAE } \\
\text { GIF (Australia) } \\
\text { JIF }\end{array}$ & $\begin{array}{l}=1.344 \\
=0.829 \\
=0.564 \\
=1.500\end{array}$ & $\begin{array}{l}\text { SIS (USA) } \\
\text { PИНЦ (Russia) } \\
\text { ESJI (KZ) } \\
\text { SJIF (Morocco) }\end{array}$ & $\begin{array}{l}=0.912 \\
=0.234 \\
=1.042 \\
=2.031\end{array}$ & $\begin{array}{l}\text { ICV (Poland) } \\
\text { PIF (India) } \\
\text { IBI (India) }\end{array}$ & $\begin{array}{l}=6.630 \\
=1.940 \\
=4.260\end{array}$ \\
\hline
\end{tabular}

\section{Введение}

Из практики известно, что синдром раздраженного кишечника (СРК) является наиболее частой разновидностью гастроэнтерологической патологии. По наблюдениям отечественных и зарубежных исследователей, симптомы этого расстройства желудочно-кишечного тракта (ЖКТ) могут наблюдаться примерно у $1 / 5$ части всего населения в разные периоды жизни [1-3]. Обычно СРК развивается в молодом возрасте и встречается у женщин почти в два раза чаще, чаще, чем у мужчин. Однако во многих случаях проявления СРК не достигают выраженного уровня клинического расстройства, требующего обращения за лечебной помощью.

Основу клиники СРК составляют расстройства стула (запор, понос, их чередование) и абдоминальные боли [1-4]. Помимо гастроэнтерологических проявлений, при СРК имеются многочисленные внекишечные проявления, в первую очередь в виде разнообразных вегетативно-сосудистых расстройств [5].

Как отмечал W.G. Thompson, из-за деликатного характера жалоб даже при наличии симптомов значительная часть пациентов стесняется обращаться к врачам, предпочитая лечиться самостоятельно дома [6]. Наблюдения показывают, что за помощью чаще всего обращаются больные СРК, страдающие выраженным болевым синдромом, и реже всего пациенты с основной жалобой на метеоризм.

Диагностика пациентов с этим расстройством ЖКТ длительное время была затруднена из-за противоречивых данных по клинике заболевания, и нечетких диагностических критериев.

\section{Материалы и методы}

На сегодняшний день разработано несколько диагностических критериев СРК. Наиболее широкое распространение получили «Римские критерии», базирующиеся на симптомологическом подходе в оценке проявления со стороны ЖКТ. В настоящее время в диагностике и лечении широко используют Римские критерии-III, не смотря на целый ряд неразрешенных вопросов и противоречий $[4,7]$.

Врачи-гастроэнтерологи в практической работе разделяют синдром раздраженного кишечника на 4 основных варианта: 1) с преобладанием запоров; 2) с преобладанием диареи; 3) с чередованием запоров и поносов; 4) проявляющийся преимущественно в виде абдоминальных болей.

Выделение разных вариантов СРК может быть полезно с практической точки зрения, так как помогает определиться с выбором тактики оптимального лечения. Следует помнить, что такое деление очень условно, поскольку у многих больных наблюдается сочетание различных симптомов и трансформация одной формы СРК в другую, что порой затрудняет определение доминирующего проявления болезни [8].

В действующей в настоящее время Международной классификации болезней десятого пересмотра (МКБ-10) термин «синдром раздраженного кишечника» закреплен сразу же в двух разделах - в психиатрической рубрике, посвященной соматоформным дисфункциям вегетативной нервной системы нижней части желудочно-кишечного тракта (шифр F45.32), а также в разделе гастроэнтерологических расстройств (шифр К58), что отражает тесные психосоматические взаимосвязи при данном заболевании ЖКТ [9].

Во многих публикациях отмечена важная роль различных психосоциальных факторов в развитии заболевания. Часть исследователей рассматривает появление клиники при СРК с системных позиций, полагая, что в основе возникновения этого функционального расстройства ЖКТ имеется разлад в единой биопсихо-социальной системе [11-17]. Так, по мнению Е.К.Баранской, СРК является биопсихосоциальным функциональным расстройством кишечника, в основе которого лежит взаимодействие двух основных механизмов: психосоциального воздействия и сенсоромоторной дисфункции (нарушения висцеральной чувствительности и двигательной активности кишечника) [14].

По данным различных исследователей, расстройства психической сферы при СРК встречаются весьма часто и могут носить полиморфный характер.

Еще в 1985 году C.Bergeron, G.Monto отмечали у пациентов с СРК ряд особенностей психического статуса, проявляющегося в виде склонности к зависимость от других, ощущении собственной неполноценности; «соматизации» с появлением симптомов неблагополучия при эмоциональных стрессах; наличия реактивной депрессии; готовности к злобным реакциям и отрицанию [18].

E.A.Walker и соавт.(1992) выявили коморбидность тревожно-депрессивных и гастроэнтерологических расстройств, a W.J.Katon и соавт.(1992) - готовность пациентов с СРК к паническим атакам [19,20]

Интерес к исследованию взаимосвязей СРК с пограничной психической патологией породил целый ряд работ, позволяющих посмотреть на данное расстройство ЖКТ, как на двойную патологию, в картине которой имеются как 
гастроэнтерологические, так

психоэмоциональные нарушения.

В исследованиях, проведенных за несколько последних десятилетий, были отмечены самые разные психические расстройства при СРК, включая невротические (в основном истероипохондрические и тревожно-фобические), аффективные и патохарактерологические нарушения [17, 21-26].

В работах В.В.Марилова и соавт. описано постепенное развитие у пациентов, страдающих психосоматическими заболеваниями ЖКТ, особого варианта приобретенного личностного расстройства - психосоматического развития личности, при котором психические расстройства, находящиеся в тесной связке с гастроэнтерологическими симптомами, не являются однородными, и поэтапно развиваются, усложняются и трансформируются [21,24,25]. В начале заболевания наблюдается этап первичной депрессивной реакции с соматизацией в ответ на острый или хронический стресс, вызвавший начало психосоматоза ЖКТ. На следующем этапе происходит закрепление затяжных психосоматических реакций с усилением тревожно-ипохондрического, фобического, истеро-невротического компонентов. Далее наблюдается наступление этапа моно- и полисистемных психосоматических циклов, который в итоге переходит в завершающий этап развития психосоматоза - психосоматическое развитие личности с выраженной психопатизацией. Типичной особенностью этого этапа является усиление прежних характерологических черт, и появление новых, ранее несвойственных для больных, в виде нарастания конфликтности и эгоцентризма.

С.В.Иванов (2000) выделил несколько вариантов сочетаний психических расстройств и гастроэнтерологической симптоматики при СРК, которые могут быть представлены органным неврозом, личностным расстройством (ипохондрическим развитием), аффективным расстройством(соматизированной депрессией) и шизофренией [27].

Ю.А.Александровский (2002) описал у больных с функциональными расстройствами ЖКТ часто встречающуюся сложную, полиморфную картину неврозоподобных состояний, преимущественно состоящую из астенических и ипохондрических расстройств, в сочетании с депрессивными, обсессивнофобическими и истерическими компонентами [23].

Б.Д.Цыганковым, И.А.Макаровой (2010) при изучении личностных акцентуаций и психологического профиля пациентов с СРК с помощью психодиагностических методик (шкала тревоги и депрессии HADS, шкала самоотчета
SCL-90, опросник Леонгарда) отмечена высокая встречаемость эмотивного типа личности, и наличие более чем у 80 \% обследованных психоэмоциональных нарушений [28].

Следует отметить, что при многолетнем опыте изучения пациентов с синдромом раздраженного кишечника и поныне нет единого мнения в отношении типичных для данного функционального расстройства ЖКТ психических расстройств.

По настоящий момент остается актуальной разработка адекватных методов терапии пограничных психических расстройств у больных с СРК, включающих в том числе и психотерапию.

Требуются дополнительные исследования, касающиеся подбора наиболее эффективных средств и методов воздействия на гастроэнтерологическую и психическую компоненты данного заболевания.

Целью работы является исследование и анализ опыта применения отечественными и зарубежными авторами психотерапевтических техник при лечении пограничных психических расстройств у пациентов с синдромом раздраженного кишечника.

\section{Результаты и обсуждение}

Несмотря на то, что многие современные авторы отмечают необходимость оказания квалифицированной психотерапевтической помощи больным СРК, до настоящего времени число работ, посвященных психотерапии при СРК, значительно уступает количеству работ, описывающих различные виды лекарственного лечения.

Лишь в небольшом числе работ описывается подробная и конкретная методология психотерапевтического процесса, Большая же часть этих публикаций ограничивается упоминанием названий примененных техник и результатам лечения. Возможно, это объясняется тем, что часть психотерапевтов, работающих с пациентами с СРК, считают, что положительный эффект в терапии часто зависит не столько от вида применяемой психотерапии, тех или иных конкретных техник, сколько от личности психотерапевта, его умения наладить сотрудничество с пациентом. В конечном итоге, основным фактором, способствующем успеху терапии, является характер складывающихся взаимоотношений между терапевтом и пациентом [29,30].

До сих пор остается актуальной рекомендация А.А. Шептулина (1997), который в начале терапии в первую очередь рекомендовал разъяснять пациентам с СРК природу имеющихся у них расстройств. В тех случаях, когда эта разъяснительная работа не была проведена, большинство больных высказывали неудовлетворение проведенным обследованием, 
требовали повторных исследований, и обращались в другие медицинские учреждения, настаивая на повторных диагностических исследованиях, будучи уверенными, что их недообследовали [9].

Е.С.Рысс отмечал, что больные с СРК весьма трудны для ведения, требуют особого внимания, нуждаются в индивидуальном подборе диеты и лечебных средств, а также включения в терапию элементов суггестии, и полезности оказания квалифицированной психотерапевтической помощи. По мнению Е.С.Рысса, только при соблюдении этих условий возможен реальный прогресс в улучшении самочувствия и качества жизни этих больных [31].

По мнению Г.А.Елизаветиной, О.Н.Минушкина (2000), не смотря на трудности лечения СРК из-за волнообразного течения и частых обострений, спровоцированных различными психоэмоциональными факторами, необходимо проявлять настойчивость, упорство, проводить терапию последовательно и терпеливо. При этом заметно уменьшается тенденция к прогрессированию, и прогноз становится благоприятным [32].

Автор одного из популярных руководств по лечению синдрома раздраженного кишечника N.Tannenhaus обращает внимание на то, что эффективность лечения значительно повышается, если пациенты с СРК принимают активное участие в излечении, изменяют стиль жизни, по возможности исключают факторы, вызывающие стресс, и овладеют аутогенной тренировкой, релаксационными приемами, и другими навыками саморегуляции, помогающими улучшить самочувствие [33].

До настоящего времени сохраняется точка зрения о том, что основной задачей психотерапии при СРК является необходимость убеждения больных в благоприятном течении болезни, предоставлении им информации о возможности длительного лечения, а также обучение вариантам психомышечной релаксации и аутотренинга. Вследствие этого в различных публикациях по проблеме лечения СРК рекомендуется дополнение лечения приемами рациональной терапии, когнитивнобихевиоральной терапии, и релаксационными методиками. Для снятия абдоминального дискомфорта и уменьшения тревожных расстройств достаточно часто прибегают к различным суггестивным методам.

В рекомендациях Российской гастроэнтерологической ассоциации и ассоциации колопроктологов (2014) по диагностике и лечению взрослых пациентов с СРК в качестве показаний для назначения психотерапевтических методов лечения указана неэффективность медикаментозного лечения продолжительностью 9-12 месяцев, с необходимостью предшествующего перед назначением психотерапии осмотра психоневролога. Отмечена возможность применения при СРК когнитивно- поведенческой терапии, гипнотерапии и психологической поддержки [34].

В.В.Марилов и соавт., а позже Е.В.Елфимова и соавт.(2015) отмечали, что при определении тактики будущей психотерапии у пациентов с психосоматическими расстройствами жКТ типы и методы психотерапии целесообразно выбирать индивидуально, в зависимости от особенностей личности, вида патологии, ее тяжести и длительности [17].

При проведении психотерапии могут быть полезными данные психодиагностики, помогающие как до начала лечения, так и в ходе его более полно оценить личностную структуру, уровни тревоги и депрессии, имеющиеся психологические проблемы $[26,35,36]$.

Ряд зарубежных и отечественных публикаций посвящен применению гипносуггестивных техник при СРК. К сожалению, приходится отметить, что количество зарубежных работ по этой теме значительно превосходит число отечественных публикации.

Еще четверть века назад рядом авторов было отмечено, что применение гипноза эффективно примерно у $80 \%$ пациентов с отчетливыми симптоматикой СРК. Особенно заметным эффект от гипносуггестивных техник был в тех случаях, когда гастроэнтерологические расстройства не сочетались с выраженными психическими расстройствами [37-41]. Помимо уменьшения абдоминальной симптоматики после гипнотических занятий, у больных на лечении гипнозом также наблюдалось снижение тревоги и повышение качества жизни [40].

В дальнейших публикациях, описывающих воздействие гипнотических техник при СРК, было подтверждено заметное уменьшение тревожно-депрессивной симптоматики после сеансов, отмечена возможность влияния на расстройства функции ЖКТ, а также эффективность гипноза для снятии абдоминальных болей у детей, подростков и взрослых [42-46]. Описан успешный опыт проведения различных вариантов гипнотических сеансов при СРК - в виде индивидуальных или групповых занятий с врачом, а также в форме самогипноза [46-47].

В работах R.H.Corney, с соавт.(1991), U.Cuntz c соавт.(1992), и более поздних публикациях описан положительный терапевтический эффект от включения приемов поведенческой психотерапии (ПТ) в лечение 
СРК [48-49]. Основой данного метода является предположение, что в основе патологических расстройств лежат нарушения поведения, которые не соответствуют требованиям среды, а ПТ сводится к коррекции неправильного поведения.

При лечении функциональных расстройств ЖКТ чаще всего используется один из самых распространенных методов ПТ - метод прогрессивной мышечной релаксации (ПМР). При применении релаксационных упражнений пациентов обучают умению расслаблять мышцы тела, дышать по брюшному типу, почувствовать во время расслабления ощушения комфорта и тепла в конечностях. Использование ПМР помогает снимать напряжение, легче переносить неприятные стрессовые ситуации, и нормализует психическое состояние пациентов.

В настоящее время при лечении больных с СРК достаточно часто применяется аутогенная тренировка (АТ), длительное время особенно популярная в качестве одного из основных методов психотерапии в нашей стране. При ее проведении последовательно применяются различные формы самовнушения, вызывающие вначале мышечное расслабление, а затем на его фоне - воздействие на нарушенные функции [50]. В гастроэнтерологической практике обычно используются как общеседативные формулы, так и формулы, направленные на снятие дискомфорта и неприятных ощущений в животе («абдоминальный» вариант аутогенной тренировки).

И.В.Пахомова, Т.А.Айвазян, В.П.Зайцев и соавт.(2008) предложили авторский модифицированный вариант абдоминальной АТ, ориентированной на лечение варианта СРК с запорами [51]. Этими же авторами проводились исследования и по сочетанному применению метода АТ и психофармакотерапии в восстановительном лечении больных с СРК, показавщие эффективность комбинированного лечения этой патологии [52].

Имеются исследования по применению метода биообратной связи для коррекции громкого урчания в животе и абдоминальных болей, при которых больные с функциональной диареей и громким урчанием в животе при регулярных занятиях учились уменьшать абдоминальный дискомфорт с помощью релаксационных методик [53,54].

Японские исследователи D. Sasaki, T. Sutoh, T. Abe (1992) также отметили положительный эффект от включения в комплексную терапию СРК методов биообратной связи, релаксационных упражнений и трансактного анализа, позволившие в итоге улучшить психоэмоциональное состояние больных и их самочувствие, эффективнее справляться с различными жизненными стрессами [55].

A.M. Van Dulmen, J.F.Fennis, G. Bleijenberg (1996) отметили эффективность при СРК долгосрочной групповой когнитивнобихевиоральной терапии, а B.Greene, E.B.Blanchard (1994), A.Payne, E.B.Blanchard (1995), и их последователи - улучшение самочувствия больных от применения когнитивной терапии [56-59].

При наличии довольно значительного количества работ, исследующих возможности применения и эффективность различных методов психотерапии при СРК, до нынешнего момента нет единой общепризнаной системы психотерапии при данном функциональном расстройстве, которая принималась бы большинством специалистов по коррекции раличных проявлений СРК. Это вызывает необходимость продолжения исследований по поиску эффективных комбинированных схем лечения с включением психотерапии, которые могут подействовать на разные системные звенья этого био-психо-социального расстройства.

В собственных исследованиях, проведенных в 2000-2016 годах, отмечено, что при проведении психотерапии целесообразно учитывать целый комплекс факторов - ведущий психопатологический синдром, особенности личности пациентов, этап течения заболевания, характер оказываемой помощи (амбулаторный, стационарный) [21,24,26,36.60-64].

В стационаре целесообразно использование техник краткосрочной психотерапии, позволяющим быстро решить текущие проблемы пациента и изменить негативное психоэмоциональное состояние. В этом плане эффективным может быть применение релаксационных техник, эриксоновского гипноза с использованием ресурсных трансов, краткосрочной позитивной терапии, нейролинвистического программирования, техник гештальт-терапии, арт-терапии. В поддерживающее лечение после выписки (особенно при запросах больных на долгосрочную глубокую работу) помимо вышеуказанных психотерапевтических методов целесообразно дополнительно включать техники системной семейной терапии, трансактного анализа и символдрамы, позволяющие как исследовать и проработать истоки существующих проблем, так и способствовать личностному росту продолжающих поддерживающую терапию пациентов [60].

В 2015 году была опубликована авторская работа по итогам применения релаксационных техник у 200 пациентов с СРК [62]. В ней описан опыт используемых для снятия беспокойства и напряжения методов психо-мышечной 
релаксации в сочетании с абдоминальным вариантом аутотренинга. Больные обучались поэтапной методике расслабления, при которой предлагалось освоить контрастные упражнения, связанные с дыханием, когда на вдохе напрягались, а на выдохе расслаблялись определенные группы мышц тела. Во время занятий постепенно формировался автоматический навык управления мышечным тонусом тела, что позволяло в дальнейшем выполнять занятия самостоятельно, уже без помощи врача. Особый акцент делался на вызывание ощущения абдоминального комфорта c помощью мягких медленных круговых поглаживающих движений области живота расслабленной ладонью, сопровождая эти действия аутосуггестией о том, что в животе все успокаивается, проходят дискомфортные ощущения. В результате применения такого подхода наблюдались как снижение тревожности и мышечного напряжения, так и общее улучшение самочувствия и существенное уменьшение (или исчезновение) гастроэнтерологической симптоматики [62].

В недавно проведенном авторском исследовании (2016), посвященном возможностям применения гипноза при комплексном лечении СРК, описаны некоторые особенности проведения гипносуггестивных сеансов, ориентированных на функциональную абдоминальную симптоматику [64].

В этом исследовании с 38 пациентами с СРК было проведено 10-15 сеансов гипноза, сфокусированных на абдоминальной симптоматике и сопутствующих психоэмоциональных проблемах. Занятия проводились по 35-45 минут трижды в неделю в течение месяца терапии, а затем велись в поддерживающем режиме с меньшей частотой. Вначале больным объяснялось, что целью сеансов является создание целительного комфортно-расслабленного состояния, способствующего ускорению процесса выздоровления. Использовались различные суггестивные приемы преимущественно недирективного характера, применяемые как в индивидуальном, так и в групповом вариантах (при палатном проведении сеансов). Во время сеансов чаще всего использовалась пошаговая четырехступенчатая модель наведения, состоящая из постановки цели, наведения трансового состояния, использования транса и переориентирования. При наведении трансовых состояний могли быть применены метафоры с неявными, косвенными намеками, показывающими возможность решения имеющихся затруднений. Так, при проведении гипноза с тревожно-депрессивными пациентами, пациентами с фобиями, при выраженном напряжении, зажатости, при спастических абдоминальных болях использовались метафоры снятия контроля, освобождение от чего-то гнетущего, облегчения. В завершении суггестии давалась установка на переход в комфортнободрствующее самочувствие Помимо этого, определенная часть суггестий проводилась с помощью «техники вставленных сообщений» в бодрствующем состоянии, когда в нейтральный по теме разговор с пациентом добавлялись специально выделенные голосом «ключевые слова» о появлении общего и абдоминального комфорта (например, слова о легкости, расслабленности, тепле, отдыхе и т.п.). Оценка эффективности терапии проводилась в динамике c помощью опросов больных, дневников самоотчета, результатов психодиагностики, проведенные в начале комбинированной терапии, и затем через месяц от начала терапии. По результатам практического использования абдоминального варианта гипноза у пациентов с синдромом раздраженного кишечника отмечена клиническая эффективность этого метода при лечении данной патологии [64].

Помимо применения вышеуказанных техник, начиная с 2001 года по настоящее время в комплексной терапии пациентов СРК был включен подход с использованием некоторых техник гештальт-терапии [60]. Следует отметить, что кроме собственных наблюдений существуют лишь единичные сообщения о возможностях применения гештальт-терапевтических техник при данной патологии. Так, в обзорной работе Я.С.Циммермана (2013) имеется ссылка на его собственный успешный опыт применения гештальт-терапии при СРК [65]. При этом достаточно высокий терапевтический потенциал гештальт-терапии при лечении психосоматических расстройств, описанный в различных руководствах [66-68], к сожалению, либо не используется в должной мере в психотерапевтических занятиях с пациентами, либо мало отражен в соответствующих публикациях.

Обобщая опыт работы с 29 пациентами с применением гештальт-техник, можно отметить, что акцент в терапевтических интервенциях в основном делался на осознавание и завершение ранее недоделанных процессов, контактов, неотреагированных эмоций, прерванных и вызывающих ощущение незавершенности ситуаций, сопровождающихся воспроизведением различных негативных переживаний. Внимание направлялось на отслеживание защитных механизмов прерывания контакта (конфлюенции, интроекции, проекции, ретрофлексии, дефлексии), при которых у пациентов терялась, рассеивалась энергия, необходимая для завершения дел. 
В работе использовались приемы с генерализацией проекций, когда пациенту предлагалось говорить от лица своей болезни, своего симптома (к примеру, тревоги или боли), на время отождествиться с ними, прояснить выгоды имеющихся расстройств, а также осознать нереализованные потребности, стоящие за имеющимся симптомом.

В ходе терапии использовалась и классическая гештальт-техника "двух стульев", при которой для пациентов создавалась возможность попеременно отождествляться с участниками прежних незавершенных психотравмирующих ситуаций, либо - провести диалог между конфликтующими частями личности.

При практической работе с пациентами применялся целый ряд творческих приемов и упражнений, описанных в классическом гештальт-подходе [66-68], включающих использование метафор, фантазий и образов, визуализаций, работу с позами и движениями, телесными упражнениями, a также других упражнений, помогающих более полному выражению заблокированных чувств, интеграции опыта.

Кроме опыта успешного применения метода гештальт-терапии, накоплены практические наблюдения по использованию у 18 пациентов с СРК техник нейро-линвистического программирования (НЛП).

Для сравнения эффективности применения техник НЛП была взята контрольная группа больных СРК, аналогичная по числу, возрасту, полу, клиническому течению заболевания. Для пациентов контрольной группы проводилась стандартная психотерапия (аутогенная тренировка, психомышечная релаксация, рациональная терапия). Длительность психотерапии в обеих группах составляла 2 месяца, за которые были проведены 20 часовых занятий. По данным психодиагностики, в результате включения техник НЛП у пациентов с СРК отмечалась положительная динамика как в психо-эмоциональной сфере, так и со стороны гастроэнтерологических проявлений, сопоставимая по эффективности с применением стандартного психотерапевтического подхода.

Терапевтическими целями при проведении психотерапии с пациентами с СРК из основной и контрольной групп являлись: уменьшение уровня напряжения; снижение личной тревожности; уменьшение ипохондрической фиксации; работа с ригидными жизненными установками; усиление личностных ресурсов и навыков управления самочувствием; работа с проблемной самооценкой; работа по снижению абдоминального дискомфорта и болей.

Из техник и упражнений нейролинвистического программирования в лечении больных преимущественно использовались: техника «хорошо сформулированная цель»; «контрастный анализ субмодальностей»; ресурсное «якорение»; «выравнивание нейрологических уровней»; «линия времени» с использованием ресурсных эпизодов; «интеграция конфликтующих частей»; «генератор нового поведения» с моделированием личностных навыков, способствующих выздоровлению, дополняемых при необходимости другими техниками НЛП [69-70].

\section{Заключение}

Основой успеха в осуществлении эффективной помощи пациентам с синдромом раздраженного кишечника является тесное сотрудничество гастроэнтерологов и психиатровпсихотерапевтов, объединяющих свои возможности в лечении этой многочисленной и сложной категории больных. До настоящего времени является актуальной тема поиска эффективных терапевтических схем при СРК.

Включение в лечение комплексного подхода, учитывающего био-психо-социальный характер СРК, позволяет увеличить эффективность терапевтического воздействия на страдающих этой патологией. Для полноценной терапии необходима оценка как гастроэнтерологических проявлений болезни, так и учет стадии заболевания, личностных особенностей, психоэмоционального статуса пациентов, позволяющих назначить более эффективные комплексные терапевтические комбинации.

Особую роль в лечении данной категории больных занимают различные психотерапевтические методы, применяемые как отдельно, так и в комбинации с психотропными препаратами с целью воздействия на проблемное психоэмоциональное состояние, основой которого являются различные проявления тревожно-депрессивного спектра. С учетом актуальности этой проблемы и необходимости ее разрешения целесообразно использовать те методы, которые уже зарекомендовали себя в качестве успешных психотерапевтических методик, а также продолжать дальнейший поиск эффективных методов воздействия на психологическую и психопатологическую составляющие данного заболевания ЖКТ. 


\begin{tabular}{l|lr|ll|ll} 
& ISRA (India) & $=\mathbf{1 . 3 4 4}$ & SIS (USA) & $=\mathbf{0 . 9 1 2}$ & ICV (Poland) & $=\mathbf{6 . 6 3 0}$ \\
Impact Factor: & ISI (Dubai, UAE) $=\mathbf{0 . 8 2 9}$ & PUHЦ (Russia) $=\mathbf{0 . 2 3 4}$ & PIF (India) & $=\mathbf{1 . 9 4 0}$ \\
& GIF (Australia) & $\mathbf{0 . 5 6 4}$ & ESJI (KZ) & $=\mathbf{1 . 0 4 2}$ & IBI (India) & $=\mathbf{4 . 2 6 0}$ \\
& JIF & $=\mathbf{1 . 5 0 0}$ & SJIF (Morocco) & $\mathbf{2 . 0 3 1}$ & & \\
\hline
\end{tabular}

\section{References:}

1. Ardatskaya MD (2010) Sindrom razdrazhennogo kishechnika: ehpidemiologiya, ehtiopatogenez, diagnostika i lechebnaya korrekciya //Farmateka. - 2010, № 13, -pp.1723.

2. Ivashkin VT, Poluehktova EA (2015) Sindrom razdrazhennogo kishechnika: patofiziologicheskie i klinicheskie aspekty problemy // Ross. zhurn. gastroehnterologii, gepatologii, koloproktologii.- 2015.- №1. - pp. 4-16.

3. Hlynov IB, Freze EB, Ryabinina OA, et al. (2016) Sindrom razdrazhennogo kishechnika: sovremennye predstavleniya o zabolevanii i perspektivy terapii // Lechashchij vrach: med. nauch.-prakt. zhurn. - 2016. - № 3. - pp. 82-85.

4. Drossman DA (2006) The Functional Gastrointestinal Disorders and the Rome III Process//Gastroenterology. -2006. -130 (5). pp.1377-1390.

5. Cygankov BD, Makarova IA (2011) Rol' vegetativnoj disregulyacii $\mathrm{V}$ razvitii vnekishechnyh proyavlenij sindroma razdrazhennogo kishechnika // Psihicheskoe zdorov'e. - 2011. - T. 9. - № 3 (58). - pp. 36-39.

6. Thompson WG (1994) Classification of functional gastrointestinal disorders.// Motility. - 1994. - №25. - pp. 10-12.

7. Sheptulin AA (2007) Rimskie kriterii III sindroma razdrazhennogo kishechnika: chto my ozhidali i chto my uvideli?//Ross. zhurn. gastroehnterologii, gepatologii, koloproktologii. -2007. -T. 17, № 2. -pp.63-68.

8. Maev IV, Cheremushkin SV (2007) Sindrom razdrazhennogo kishechnika. Rimskie kriterii III // Gastroehnterologiya. (prilozhenie k zhurnalu Consilium Medicum).- 2007.- №1.pp. 29-33

9. Sheptulin AA (1997) Sindrom razdrazhennoj kishki: sovremennye predstavleniya o patogeneze, klinike, diagnostike, lechenii// Ross. zhurn. gastroehnterologii, gepatologii, koloproktologii. -1997, №5. - pp. 88-90.

10. (2005) Mezhdunarodnaya klassifikaciya boleznej MKB-10/ISD-10 / Per.s angl. M.:, World Health Organization -1995.- T.1 (1). $698 \mathrm{p}$.

11. Smulevich AB (1997) Psihosomaticheskie rasstrojstva// Soc.klin.psihiatriya -1997. -№ 1. pp. 5-18.

12. Goldberg J, Davidson P (1997) A biopsychosocial understanding of the irritable bowel syndrome: a review.// Can J Psychiatry. Oct 1997; Vol 42. -pp.835-840.
13. Drossman DA (1998) Presidental Address: Gastrointestinal illness and Biopsychosocial Model. Psychosom Med. 1998; №60: -pp. 258-267.

14. Baranskaya EK (2000) Sindrom razdrazhennogo kishechnika: diagnostika i lechenie // Consilium medicum. - 2000. - T.II. - №7. - pp. 287-292.

15. Ivashkin VT, Poluehktova EA, Belkhusht S (2003) Sindrom razdrazhennogo kishechnika kak biopsikhosotsial'noe zabolevanie // Klinicheskie perspektivy $\mathrm{v}$ gastroehnterologii, gepatologii. - 2003. - N 6 . - pp. 2-10.

16. Makhov VM, Romasenko LV, Turko TV, et al. (2013) Mezhdistsiplinarnyj podkhod k sindromu razdrazhennogo kishechnika // Russkij meditsinskij zhurnal 2013. T.21, №13. - pp.702-704

17. Elfimova EV, Elfimov MA, Berezkin AS (2015) Psikhosomaticheskie vzaimootnosheniya pri zabolevaniyakh zheludochno-kishechnogo trakta na modeli sindroma razdrazhennogo kishechnika // Ehksperimental'naya i klin. gastroehnterologiya. 2015. №4 (116) . - pp. 8388.

18. Bergeron CM, Monto GL (1985) Personality patterns seen in irritable bowel syndrome patients. //Amer. J. Gastroenterol. -1985.- Vol. 80, №6.- pp.448-451.

19. Walker EA, Katon WJ, Jemelka RP, et al. (1992) Comorbidity of gastrointestinal complaints, depression, and anxiety in the Epidemiologic Catchment Area (ECA) Study // Am-JMed., 1992, Jan 24; 92(1A) - pp.26-30.

20. Katon WJ, Von Korff M, Lin E (1992) Panic disorder relationship to high medical utilization.//Am-J-Med. 1992 Jan 24; 92(1A) pp.7-11.

21. Marilov VV, Korkina MV, Esaulov VI (2000) Lichnostnye osobennosti i kharakter psikhicheskikh narushenij pri sindrome razdrazheniya tolstoj kishki // Sotsial'naya i klinicheskaya psikhiatriya. - 2000. - T. 10, № 4. - pp. 21-27.

22. Aleksandrovskij YA (2002) Pogranichnye psikhicheskie rasstrojstva pri somaticheskikh zabolevaniyakh // Psikhiatriya i psikhofarmakoterapiya. - 2002. T. 4, №1, - pp. 4-7.

23. Roganov DA (2005) Affektivnye rasstrojstva pri sindrome razdrazhennogo kishechnika (klinicheskie osobennosti, diagnostika, lechenie) : avtoref. dis ... kand. med. nauk. Moscow, 2005. - 25 p. 
24. Marilov VV, Esaulov VI (2000) Psihicheskie narusheniya pri sindrome vozbudimoj tolstoj kishki//Vestnik RUDN. Seriya: Medicina. 2000. -№ 3. -pp. 98-101.

25. Marilov VV, Orestova TY, Marilova TY (2005) Psikhopatologicheskie varianty sindroma razdrazhennoj tolstoj kishki // Zhurnal nevrologii i psikhiatrii. -2005 - T.105. №6. pp.64-65.

26. Esaulov VI (2016) The experience of using psychodiagnostic methods on patient with irritable bowel syndrome //Voprosy transformatsii obrazovaniya (SCIEURO, London). - 2016. №1. - pp.238-250.

27. Ivanov SI (2000) Organnye nevrozy. // V kn. Pogranichnaya psikhicheskaya patologiya $\mathrm{v}$ obshhemeditsinskoj praktike, (pod red. A.B.Smulevicha), M.: Izdatel'skij dom Russkij vrach. - 2000, - pp.81-89.

28. Cygankov BD, Makarova IA (2010) Lichnostnye akcentuacii i psihologicheskij profil' pacientov s sindromom razdrazhyonnogo kishechnika. - //Vestnik nevrologii, psihiatrii i nejrohirurgii. - 2010. - №12. - pp.35-39.

29. Owens DM, Nelson DK, Talley NJ (1995) The irritable bowel syndrome: long-term prognosis and the physician-patient interaction.// Ann Intern Med 1995 Jan 15;122(2) - pp.107-112.

30. Van Dulmen AM, Fennis JF, Mokkink HG, et al. (1995) Doctor-dependent changes in complaint-related cognitions and anxiety during medical consultations in functional abdominal complaints.// Psychol Med. - 1995. Sep; 25 (5): - pp.1011-1018.

31. Ryss ES (1998) Nekotorye aktual'nye voprosy ehnterologii // Mir mediciny.-1998. -№4.pp.15-19.

32. Elizavetina GA, Minushkin ON (2000) Lechenie sindroma razdrazhennogo kishechnika sovremennymi preparatami// Kremlevskaya medicina. Klinicheskij vestnik. - 2000- № 1, pp. 74-78.

33. Tannenhaus N (1995) Hronicheskij sindrom razdrazhennoj tolstoj kishki./Per.s angl. Moscow: KRON-PRESS, -1995. - 144 p.

34. Ivashkin VT, Shelygin YuA, Baranskaya EK, et al. (2014) Klinicheskie rekomendacii Rossijskoj gastroehnterologicheskoj associacii, Associacii koloproktologov Rossii po diagnostike i lecheniyu bol'nyh s sindromom razdrazhennogo kishechnika// Ross. zhurn. gastroehnterologii, gepatologii, koloproktologii. - 2014 № 2. - pp. 92-101.

35. Kozlova IV, Myalina YN, Lekareva LI, et al. (2014) Psihologicheskie osobennosti pacientov s funkcional'nymi i vospalitel'nymi zabolevaniyami kishechnika // Saratovskij nauchno-medicinskij zhurnal. - 2014. - T. 10, № $1 .-$ pp. $80-85$.
36. Esaulov VI (2015) O nekotoryh ehmocional'nolichnostnyh harakteristikah pacientov $\mathrm{s}$ sindromom razdrazhennogo kishechnika// European research. -2015. -№ 5 (6). -pp.76-78.

37. Whorwell PJ, Prior A, Faragher EB (1984) Controlled trial of hypnotherapy in the treatment of severe refractory irritablebowel syndrome // Lancet. 1984. №2. -pp.1232-1234.

38. Whorwell PJ (1991) Use of hypnotherapy in gastrointestinal disease // Br. J. Hosp. Med. 1991. Vol.45. №1. - pp.27-29.

39. Whorwell PJ, Houghton LA, Taylor EE, et al. (1992) Physiological effects of emotion: assessment via hypnosis. // Lancet -1992. Jul 11;340 (8811). - pp. 69-72

40. Houghton LA, Heyman DJ, Whorwell PJ (1996) Symptomatology, quality of life and economic features of irritable bowel syndrome the effect of hypnotherapy.// Alimentary pharmacology \& therapeutics. -1996, №10. pp.91-95.

41. Palsson OS, Burnett CK, Meyer K, et al. (1997) Hypnosis treatment for irritable bowel syndrome. Effects on symptoms, pain threshold and muscle tone.// Gastroenterology, 112, A803.

42. Houghton LA, Larder S, Lee R, et al. (1999) Gut focused hypnotherapy normalizes rectal hypersensitivity in patients with irritable bowel syndrome (IBS). Gastroenterology, -1999.- 116: A1009.

43. Whorwell P (2005) Review article: the history of hypnotherapy and its role in the irritable bowel syndrome.//Alimentary Pharmacology \& Therapeutics. -2005. - № 22. - pp. 1061-1067.

44. Gottsegen D (2011) Hypnosis for functional abdominal pain // Am. J. Clin. Hypn. -2011. Vol.54. №1. pp.56-69.

45. Vlieger AM, Rutten JM, Govers AM, et al. (2012) Long-term follow-up of gut-directed hypnotherapy vs. standard care in children with functional abdominal pain or irritable bowel syndrome // Am. J. Gastroenterol. -2012. Vol.107. №4. pp.627-631. doi: 10.1038/ajg.2011.487.

46. Anbar RD (2001) Self-hypnosis for the treatment of functional abdominal pain in childhood // Clin. Pediatr. -2001. Vol.40. №8. pp.447-451.

47. Harvey RF, Hinton RA, Gunary RM, et al. (1981) Individual and group hypnotherapy in treatment of refractory irritable bowel syndrome // Lancet. -1989. №1. pp.424-425.

48. Corney RH, Stanton R, Newell R, et al. (1991) Behavioural psychotherapy in the treatment of irritable bowel syndrome.// J Psychosom Res. 1991; 35(4-5)- pp.461-469.

49. Cuntz U, Pollmann H, Enck P (1992) Behavior therapy in gastrointestinal functional 


\begin{tabular}{|c|c|c|c|c|c|c|}
\hline Impact Factor: & $\begin{array}{l}\text { ISRA (India) } \\
\text { ISI (Dubai, UAE } \\
\text { GIF (Australia) } \\
\text { JIF }\end{array}$ & $\begin{array}{l}=1.344 \\
=0.829 \\
=0.564 \\
=1.500\end{array}$ & $\begin{array}{l}\text { SIS (USA) } \\
\text { PИНЦ (Russia) } \\
\text { ESJI (KZ) } \\
\text { SJIF (Morocco) }\end{array}$ & $\begin{array}{l}=0.912 \\
=0.234 \\
=1.042 \\
=2.031\end{array}$ & $\begin{array}{l}\text { ICV (Poland) } \\
\text { PIF (India) } \\
\text { IBI (India) }\end{array}$ & $\begin{array}{l}=6.630 \\
=1.940 \\
=4.260\end{array}$ \\
\hline
\end{tabular}

disorders.// Z-Gastroenterol. 1992 Jan; 30(1): pp.24-34.

50. Filatov AS (1979) Autogennaya trenirovka. Kiev, Zdorov'ya. -1979. - 144 p.

51. Pahomova, IV, Ajvazyan TA, Zajcev VP, et al. (2008) Autogennaya trenirovka v vosstanovitel'nom lechenii bol'nyh s sindromom razdrazhennogo kishechnika s preobladaniem zaporov // Voprosy kurortologii, fizioterapii i lechebnoj fizicheskoj kul'tury. - 2008. - №1. pp.24-27.

52. Pahomova IV, Ajvazyan TA, Zajcev VP, et al. (2008) Ehffektivnost' sochetannogo primeneniya autogennoj trenirovki i psihofarmakoterapii $\mathrm{v}$ vosstanovitel'nom lechenii bol'nyh sindromom razdrazhennogo kishechnika s preobladaniem zaporov //Vestnik vosstanovitel'noj mediciny. -2008. № 2. - pp. 55-58.

53. Latimer PB (1981) Biofeedback and behavioral approaches to disorders of gastrointestinal tract.// Psychother.psychosom. 1981. - Vol. 36. - pp. 200-212.

54. Whitehead WE (1992) Biofeedback treatment of gastrointestinal disorders.// BiofeedbackSelf-Regul. -1992 Mar; 17(1) - pp.59-76.

55. Sasaki D, Sutoh T, Abe T (1992) Psychosomatic treatment of irritable bowel syndrome. // Nippon Rinsho , 1992. Nov.; 50 (11), - pp. 2758-2763.

56. Van Dulmen AM, Fennis JF, Bleijenberg G (1996) Cognitive-behavioral group therapy for irritable bowel syndrome: effects and long-term follow-up.// Psychosom Med. -1996; 58: pp.508-514.

57. Greene B, Blanchard EB (1994) Cognitive therapy for irritable bowel syndrome.// JConsult-Clin-Psychol. 1994 Jun; 62(3); pp.576-82.

58. Payne A, Blanchard EB (1995) A controlled comparison of cognitive therapy and self-help support groups in the treatment of irritable bowel syndrome.// J Consult Clin Psychol. 1995;63: -pp.779-786.

59. Li L, Lishou X, Schenghong Z, et al. (2014) Cognitive-behavioral therapy for irritable bowel syndrome : A meta-analysis // Journal of
Psychosomatic Research. -2014 July.Vol. 77. Issue 1. - pp. 1-12. DOI: dx.doi.org /10/1016/j.jpsychores.2014.03.006

60. Esaulov VI, Marilov VV (2001) Kompleksnaya psihoterapiya sindroma razdrazhennogo kishechnika//Vestnik RUDN. Seriya: Medicina. - 2001. -№ 2. -pp.54-57.

61. Esaulov VI (2015) O dinamike psihoehmocional'nogo sostoyaniya u pacientok s bolevoj formoj sindroma razdrazhennogo kishechnika pri psihoterapii // Sovremennye problemy nauki i obrazovaniya. - 2015. - № 6 . DOI : $10.17513 /$ spno.23650

62. Esaulov VI (2015) Ob opyte primeneniya relaksacionnyh metodov $\mathrm{v}$ lechenii pacientov $\mathrm{s}$ sindromom razdrazhennogo kishechnika // Vestnik nevrologii, psihiatrii, nejrohirurgii 2015. № 10. -pp. 60-64

63. Esaulov VI (2016) Ob opyte primeneniya u bol'nyh $\mathrm{s}$ sindromom razdrazhennogo kishechnika metodov kompleksnoj psihodiagnostiki //Sovremennye problemy nauki i obrazovaniya. - 2016. - № 4. DOI:10.17513/spno.24911

64. Esaulov VI (2016) Primenenie abdominal'nogo varianta gipnoza $\mathrm{v}$ kompleksnom lechenii sindroma razdrazhennogo kishechnika // Vestn. Novg. gos. un-ta. Ser.: Medicinskie nauki. 2016. № 1(92). -pp.90-94.

65. Cimmerman YS (2014) Sindrom razdrazhennoj kishki : kakova ego istinnaya sushchnost' ? // Klinicheskaya medicina - 2014. №7. -pp.19-29.

66. Romanenko OK (1995) Prakticheskaya geshtal'tterapiya. M.:, Folium. 1995- 112 p.

67. Perlz F, Gudmen P, Hefferlin R (2001) Praktikum po geshtal't-terapii - Per.s angl. M.:Izd-vo Instituta Psihoterapii.2001. - $-240 \mathrm{p}$.

68. Bulyubash ID (2004) Rukovodstvo po geshtal'tterapii. M.: Izd-vo Instituta Psihoterapii. -2004.$768 \mathrm{p}$.

69. Kovalev SV (2006) Iscelenie s pomoshch'yu NLP. M.: Izd-vo: KSP+. -2006. - 542 p.

70. Gordeev MN, Gordeeva EG (2008) NLP v psihoterapii. M.; Izd-vo:Psihoterapiya. -2008. $272 \mathrm{p}$. 